\title{
Molecular characterization of breast cancer cell lines by clinical immunohistochemical markers
}

\author{
ANDRÉ DE LIMA MOTA ${ }^{1}$, ADRIANE FEIJO EVANGELISTA ${ }^{1}$, \\ TACIANE MACEDO $^{1}$, RENATO OLIVEIRA ${ }^{1}$, CRISTOVAM SCAPULATEMPO-NETO ${ }^{1,2}$, \\ RENÉ ALOISIO DA COSTA VIEIRA ${ }^{3}$ and MARCIA MARIA CHIQUITELLI MARQUES ${ }^{1,4}$ \\ ${ }^{1}$ Molecular Oncology Research Center; Departments of ${ }^{2}$ Pathology and ${ }^{3}$ Mastology and Breast Reconstruction, \\ Barretos Cancer Hospital, Barretos, São Paulo 14.784-400; ${ }^{4}$ Barretos School of Health Sciences (FACISB), \\ Barretos, São Paulo 14.785-002, Brazil
}

Received March 31, 2016; Accepted December 21, 2016

DOI: $10.3892 / 01.2017 .6093$

\begin{abstract}
Breast cancer is the leading cause of cancer mortality in females worldwide. Studies based on gene expression profiles have identified different breast cancer molecular subtypes, such as luminal A and B cells, cancer cells that are estrogen receptor (ER) and/or progesterone receptor (PR) positive, human epidermal growth factor 2 (HER2)-enriched cells, cancer cells that exhibit an overexpression of the oncogene $H E R 2$, and triple-negative cells, cancer cells that are negative for ER, PR and HER2 expression. Immunohistochemistry is the most common type of method used for the identification of these molecular subtypes, through the identification of specific cell receptors. The present study aimed to evaluate the ER, $\mathrm{PR}$ and HER2 receptor expression in human breast cancer cell lines, and to classify the corresponding molecular subtype comparing two alternative methods. In the present study, a panel of human mammary carcinoma cell lines: BT-20; Hs578T; MCF-7; MCF-7/AZ; MDA-MB-231; MDA-MB-468; SKBR3; and T47D were used. Immunohistochemical and immunocytochemistry assays were used to characterize the breast cancer subtypes of these cell lines according to the expression of ER, PR and HER2 receptors. The results revealed the molecular characterization of this panel of breast cancer cell lines, using the differential expression of classical and clinically used markers in concordance with previous studies. In addition, these data are important for additional in vitro studies of these specific receptors.
\end{abstract}

Correspondence to: Dr Marcia Maria Chiquitelli Marques, Molecular Oncology Research Center, Barretos Cancer Hospital, 1331 Av. Antenor Duarte Vilela, Barretos, São Paulo 14.784-400, Brazil

E-mail: mmcmsilveira@gmail.com

Key words: breast cancer, breast cancer cell lines, immunocytochemistry, immunohistochemistry, cell receptors

\section{Introduction}

Breast cancer is the second most common type of malignant neoplasm worldwide, and the most common type amongst females (1). The incidence of breast cancer in Brazil is similar to the incidence in developed countries, and varies according the regions, with the highest rates in southern region (2). A previous study demonstrated a decrease in female breast cancer mortality rates in the majority of developed Brazilian states, possibly due to an improvement of healthcare in these regions (3).

Despite advances in breast cancer clinics and research concerning care management, several questions remain. Breast tumors with similar histopathological appearances may exhibit different clinical presentations, levels of disease aggression and treatment responsiveness. The heterogeneity in breast cancer cell phenotypes and plasticity of the tumor microenvironment affects the therapeutic response and disease progression $(4,5)$. At present, treatment options are based on the characteristics of the particular tumor, and treatment is multimodal (6-8). Therefore, a molecular approach by immunohistochemical evaluation is necessary to identify breast cancer subtypes for further treatment options $(4,5)$.

Perou et al (9) initially classified breast tumors into four molecular subtypes according to their gene expression profiles: Estrogen receptor $(\mathrm{ER})^{+} /$luminal-like, receptor tyrosine-protein kinase erbB-2 (Erb-B2), basal-like and normal breast tissue. At present, due to the limitations of array analysis in clinical practice, particularly the high costs of this method, classification protocols have incorporated immunohistochemical classification techniques in clinical practice (10). There is clinical interest in distinguishing breast cancer subtypes, particularly the most aggressive types that exhibit poor prognosis, which possess a triple-negative profile that does not exhibit ER, progesterone receptors (PR) or the oncogene HER2 (11-13). The differentiation of luminal B cells, which exhibit higher proliferation rates, from luminal A subtypes has a direct effect on the selection of treatment strategy $(12,13)$. PR expression in $>20 \%$ of tumor cells was suggested to increase luminal A sensibility (14), and the initial cut-off of the level of proliferation marker protein KI67 at $\geq 14 \%$ (10) that was initially proposed has been revealed to be increased to $\geq 20 \%$, but this has not been confirmed (15). 
Cancer cells in vitro are immortal and may exhibit spontaneously modified characteristics, so it is important to regularly characterize them. Although there are strong associations between particular types of cell line and specific immunohistochemical characteristics, they are not predictive and therefore it is important to translate in vitro data to a clinical context (16). The present study aimed to characterize the cell phenotypes of a panel of human breast carcinoma cell lines by measuring the expression levels of the markers classically used in clinic: ER, PR, HER2 and KI67, using IHC and immunocytochemistry (ICC). This data will be useful for subsequent in vitro studies investigating the expression of the specific receptors of the markers.

\section{Materials and methods}

Breast cancer cell lines. A total of 8 breast cancer cell (BCC) lines were used in the present study: BT-20; Hs578T; MCF-7; MCF-7/AZ; MDA-MB-231; MDA-MB-468; SKBR3; and T47D were maintained in Dulbecco's modified Eagle's medium (Gibco; Thermo Fisher Scientific, Inc., Waltham, MA, USA) with $10 \%$ fetal bovine serum (Gibco; Thermo Fisher Scientific, Inc.) and $1 \%$ penicillin and streptomycin (Gibco; Thermo Fisher Scientific, Inc.) in a $5 \% \mathrm{CO}_{2}$ atmosphere at $37^{\circ} \mathrm{C}$. The cells were provided by Dr Rui M. V. Reis from Molecular Oncology Research Center at Barretos Cancer Hospital (Barretos, Brazil).

IHC and ICC assays. The IHC assays were performed using paraffin-embedded cell pellets obtained from $150 \mathrm{~cm}^{2}$ flasks, fixed in $10 \%$ buffered formalin. The cell pellet was centrifuged at $1.027 \mathrm{x} \mathrm{g}$ for $5 \mathrm{~min}$ at room temperature, dehydrated in different concentrations of ethanol bath (50, 70, 95 and 100\%), immersed in xylene baths for $2 \mathrm{~min}$, and embedded in paraffin blocks to obtain $4-\mu \mathrm{m}$ sections. The IHC process followed the standard automated process of the Ventana BenchMark Ultra automated slide staining system (Ventana Medical Systems, Inc., Tucson, AZ) using a ultraView 3,3'-diaminobenzidine (DAB) detection kit and prediluted primary antibodies from Ventana Medical Systems, Inc. with the following specificities: ER (cat. no., 790-4325; clone SP1), PR (cat. no., 790-2223; clone 1E2), HER2 (cat. no., 792991; clone 4B5) and Ki67 (cat. no., 790-4286; clone SP6).

ICC was performed as follows: The cells were seeded onto circular glass coverslips of $24 \mathrm{~mm}$ diameter at $80 \%$ confluence, fixed in 4\% paraformaldehyde (Merck KGaA, Darmstadt, Germany) for $15 \mathrm{~min}$ at $4^{\circ} \mathrm{C}$, followed by permeabilization with $0.2 \%$ Triton X-100 for 4 min. Coverslips were placed on the slides, which were treated with $3 \%$ hydrogen peroxide in methanol to block endogenous peroxidase and incubated in a humidified chamber for $60 \mathrm{~min}$ at room temperature. Anti-ER, anti-PR and anti-HER 2 were used as the primary antibodies using pre-dilution solutions as aforementioned. The slides were washed with PBS and incubated for $30 \mathrm{~min}$ at room temperature with the polyvalent secondary antibody from the UltraVision Quanto Detection System horseradish peroxidase DAB kit (cat. no. TL-125-QHD; Thermo Fisher Scientific, Inc.) and streptavidin-peroxidase (Thermo Fisher Scientific, Inc.) for 10 min using a DAB chromogenic kit (Agilent Technologies, Inc., Santa Clara, CA, USA).
The ER and PR staining reactions were scored according to nuclear intensity, weak, moderate and high, and extension through the following criteria: $0 \%$, negative; 1 to $25 \%$, low expression; 26 to $50 \%$, moderate expression; 51 to $75 \%$, high expression; above $>75 \%$, very high expression. HER 2 expression was analyzed according to the American Society of Clinical Oncology (ASCO) /College of American Pathologists HER2 test guidelines (17).

\section{Results}

The expression level of specific receptors in the BCC BT-20, Hs578T, MCF-7, MCF-7/AZ, MDA-MB-231, MDA-MB-468, SKBR3 and T47D lines, was assessed by IHC (Fig. 1) and ICC (Fig. 2). Despite differences with respect to expression intensity between the two techniques, similar results regarding ER, PR and HER2 expression were observed.

The staining intensity of the cell lines tested using IHC is represented in Fig. 3, which revealed the BCC subtype characterizations as i) MCF-7, MCF7-AZ and T47D, Luminal; ii) BT-20 and SK-BR3, HER2 overexpressed and; iii) MDA-MB-231, MDA-MB-468 and Hs578T, triple negative subtype.

\section{Discussion}

Breast cancer is a heterogeneous disease characterized by significant variability in morphological and pathological features that may exhibit differences regarding therapeutic responses and disease progression, which significantly affect the management of this disease (5). Perou et al (9) initially classified breast tumors into four molecular subtypes according to their gene expression profiles: $\mathrm{ER}^{+} /$luminal-like, HER2, basal-like and normal breast. This molecular classification has been altered to five categories: Luminal A, Luminal $\mathrm{B}+\mathrm{C}$, normal-like, basal like and ErbB2 $2^{+}$, based on prognostic evaluation (18). Recently a novel subtype classified as claudin-low has also been identified based on microarray gene expression platform (19).

IHC has been applied as a diagnostic method to identify the five classical subtypes of breast cancer based on immunohistochemical profiles: Luminal $\mathrm{A}, \mathrm{ER}^{+}$and/or progesterone receptor positive, HER2-; luminal B, ER ${ }^{+}$and or $\mathrm{PR}^{+}, \mathrm{HER}^{+}$; basal-like, ER-PR-HER2- ${ }^{-}$, cytokeratin $5 / 6^{+}$, and/or HER1 ${ }^{+}$; HER2 ${ }^{+} / \mathrm{ER}^{-}$, ER-PR ${ }^{-}$and HER2 ${ }^{+}$; and unclassified, negative for all five markers. A strong correlation was observed between the molecular marker profiles and immunohistochemical evaluation (20). HER2 tumors are immunohistochemically categorized as $+++/+++$ or when fluorescence in situ hybridization demonstrates HER2 gene amplification $(17,21)$. The most recent ASCO/CAP recommendation for HER2 testing changed the threshold for determining HER2-positive status with immunohistochemistry to strong circumferential membranous staining in $\geq 30 \%$ of the tumor cells, whereas the previous threshold was $\geq 10 \%$ (17), which may cause discrepancies between studies. The Ki67 index was added to immunohistochemical evaluation, and the labeling index of $13.25 \%$ separates luminal A/HER 2- and luminal B/HER - tumors, with a sensitivity and specificity of 72 and $77 \%$, respectively, for gene expression (22). As it is not cost-effective to perform individual 


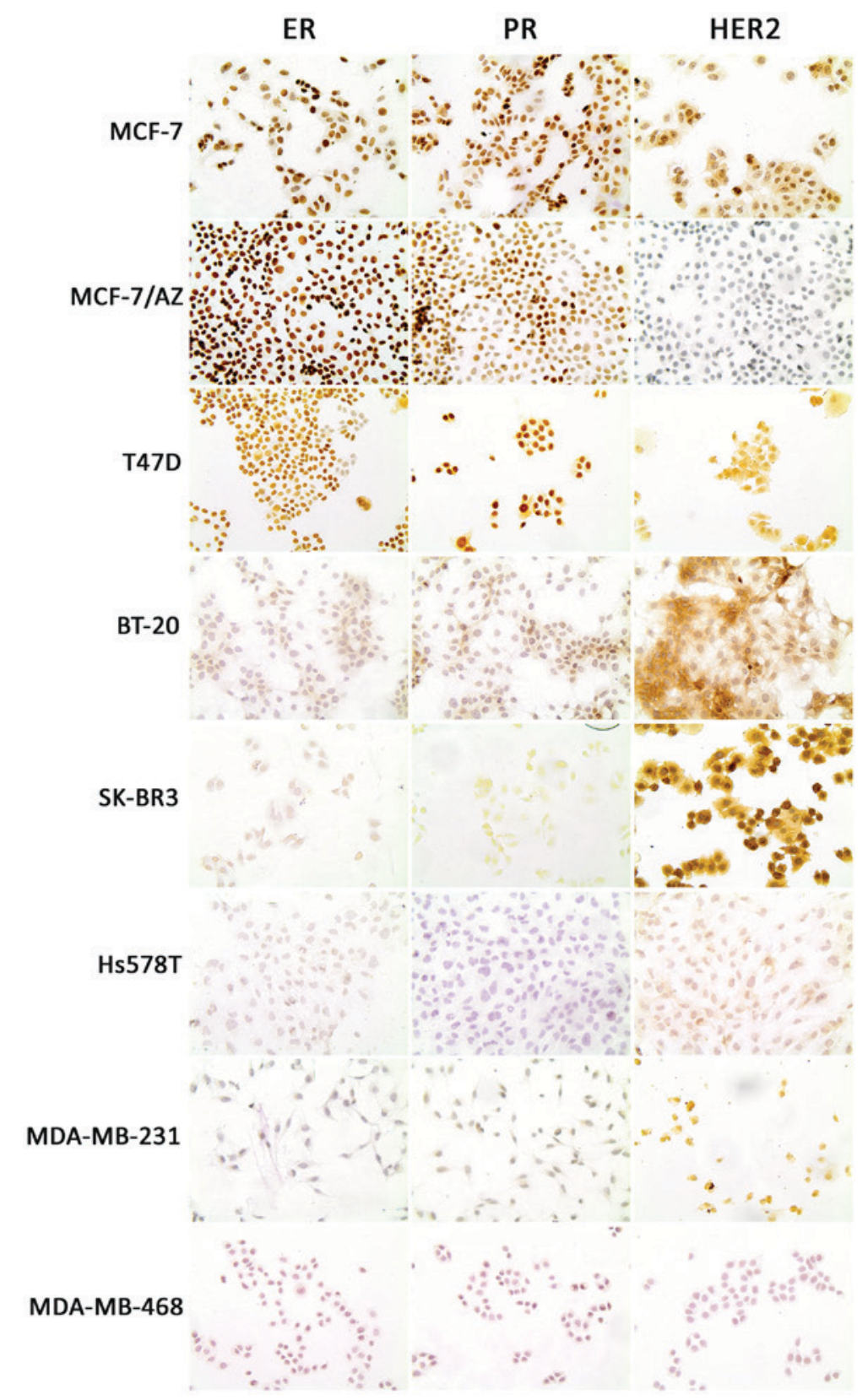

Figure 1. Representative immunohistochemistry for ER, PR, and HER2 in breast cancer cell lines MCF-7, MCF-7/AZ, T47D, BT-20, SKBR3, Hs578T, MDA-MB-231 and MDA-MB-468. Scale bar=100 $\mu$ M. ER, estrogen receptor; PR, progesterone receptor; HER2, human epidermal growth factor.

molecular classifications in clinical practice, the 12 th $\mathrm{St}$ Gallen Consensus (10) was adopted, and comprises 5 classifications based on immunohistochemical results: Luminal A, Ki67 <14; Luminal B HER ${ }^{-}$Ki67 $\geq 14$; Luminal B Her ${ }^{+}$; HER2 positive, non-luminal and basal-like, triple-negative. Prat et al (14) suggested the inclusion of PR positive tumor cells $\geq 20 \%$ to increase luminal A sensitivity Novel amendments to classification protocols have been proposed to include the level of KI67 expression: High expression, $\geq 20 \%$; intermediate expression, 14-19\%; PR negative or low expression, $<20 \%$, but this has not been formally adopted (15). The present study aimed to characterize ER, PR, Ki67 and HER 2 receptor expression in BCC lines to classify the corresponding molecular subtype (10).

According to the gene expression profiles, breast tumors may be classified into luminal A,luminalB and HER2-overexpressed and triple-negative subtypes (9). The expense of this classification system has limited the incorporation of gene expression profiling into clinical practice, and therefore, IHC assays are used for the identification of molecular subtypes, which may characterize the gene expression profiles of the cells through ER, PR and HER2 receptors (13). The differences observed in the marker intensity between the two techniques did not affect the classification of the molecular subtypes of the cell lines.

The classification of BCC lines includes the presence of ER on luminal A and B, HER2 overexpression of HER2 oncogene on HER 2 subtype, and absence of ER expression, PR and HER2 on triple-negative cells. Although certain luminal B tumors may be identified by their expression of HER2, the distinction between luminal A and $\mathrm{B}$ relies on the observation of the rate of proliferation, including the level of expression of Ki67 in the luminal B subtype $(9,12,23)$. In the present study, 


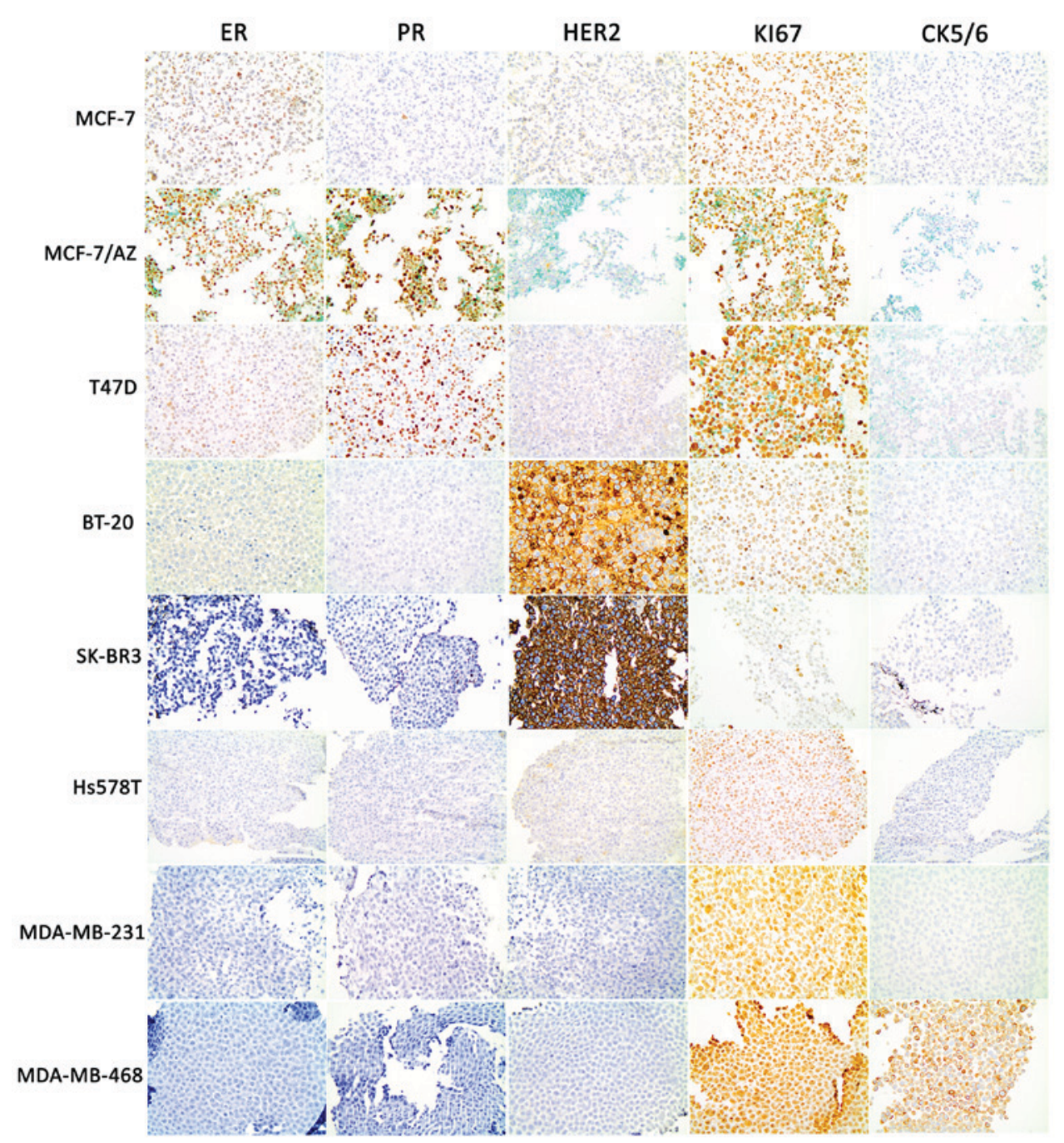

Figure 2. Representative immunocytochemistry for ER, PR, HER2, KI67 and CK5/6 in breast cancer cell lines, MCF-7, MCF-7/AZ, T47D, BT-20, SKBR3, Hs578T, MDA-MB-231 and MDA-MB-468. Scale bar=100 $\mu$ M. ER, estrogen receptor; PR, progesterone receptor; HER2, human epidermal growth factor; CK, cytokeratin.

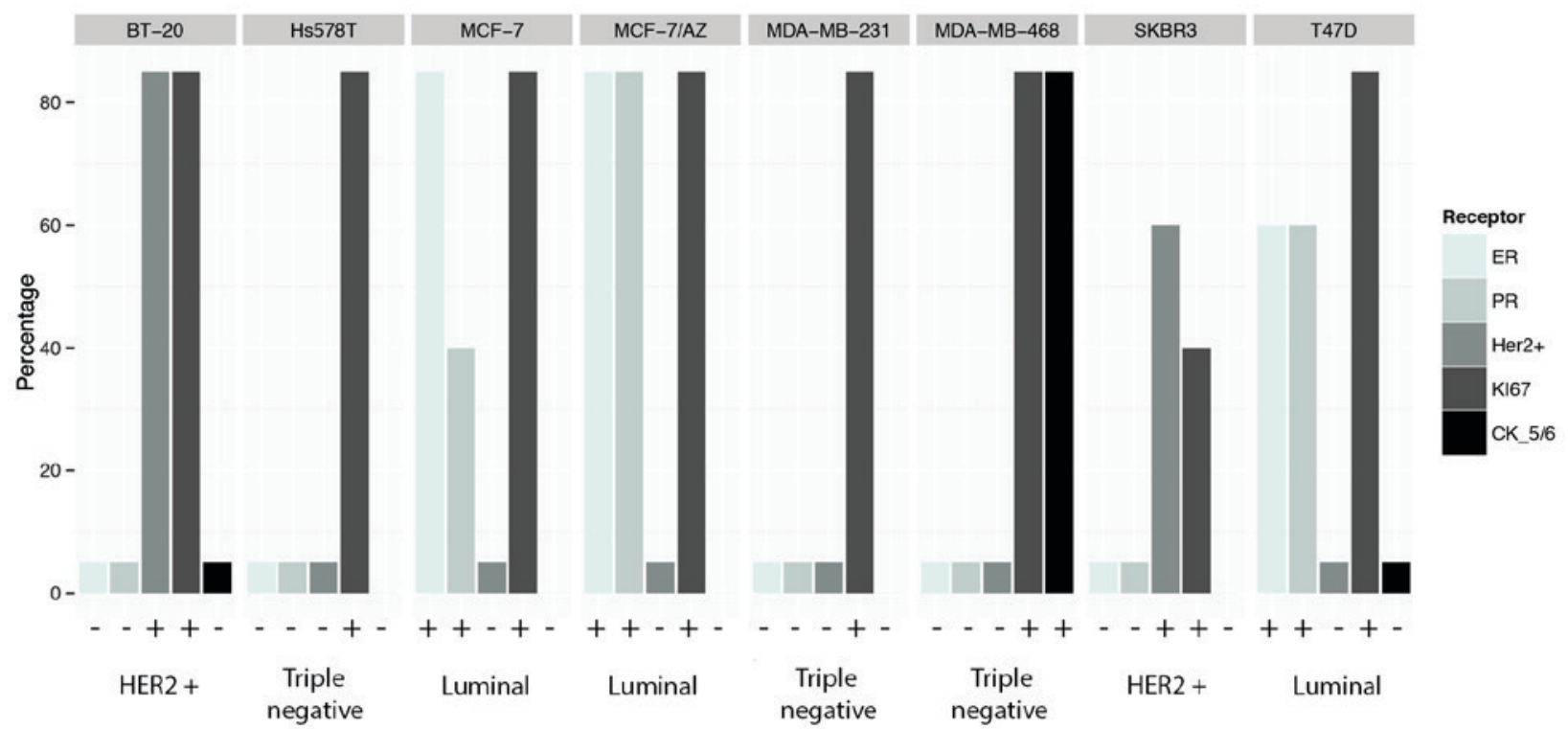

Figure 3. Staining intensity scores of receptor markers ER, PR, HER2, Ki67 and CK5/6 expression, obtained by immunohistochemistry and immunocytochemistry in breast cancer cell lines BT-20, Hs578T, MCF-7, MCF-7A/Z, MDA-MB-231, MDA-MB-468, SKBR3 and T47D. Luminal, ER+/PR ; HER2 ${ }^{+}$, HER2 overexpressed; triple-negative, does not exhibit ER, PR or HER2. ER, estrogen receptor; PR, progesterone receptor; CK, cytokeratin; HER2, human epidermal growth factor. 
it was not possible to observe a difference between luminal A and $\mathrm{B}$, possibly due to changes in the levels of expression of some proteins observed on longer culture conditions. However, the general classification is in concordance with American Type Culture Collection and associated studies (24).

Subik et al (16) performed an IHC analysis of 17 breast cancer cell lines, using ER, PR, HER2, cytokeratin (CK) 5/6, cytokine and growth factor (CGFR), Ki-67 and androgen receptor (AR) as markers, although did $\mathrm{Ki}-67$ and $\mathrm{AR}$ were not used for the classification, and tumors were considered HER2-positive only when this protein was highly expressed. The authors used the Carey initial classification (20), and observed a high level of $\mathrm{Ki}-67$ in this panel of breast cancer cell lines. The aforementioned study considered the importance of evaluating the in vitro cell characteristics, but did not compare the molecular classification with the final results observed in the immunohistochemistry analysis.

BCC lines are used as in vitro models to investigate gene and protein expression, responses to drugs and for toxicity assessments. These cells must be characterized for quality control, and to provide better reproducibility of studies based on the expression of specific receptors. The results of the present study demonstrate that the characterization of the molecular subtypes of BCC lines by IHC and ICC assays is possible, but that there is not complete concordance, suggesting there is a potential limitation on the immunohistochemistry classification or a false-negative result.

In conclusion, the present study characterized the phenotype of a panel of BCC lines, according to their ER, PR and HER2 markers. This data may provide a tool for an increased understanding of cellular behavior, and confirm knowledge from prior in vitro studies based on cell receptor expression. The present study demonstrates similarities between ICC and IHC techniques, which may affect local quality control protocols for these cells.

\section{Acknowledgements}

The present study was partially supported by FINEP (grant no. MCTI/FINEP/MS/SCTIE/DECIT01/2013-FPXII-BIOPLAT) and by Barretos Cancer Hospital Incentive to research fund.

\section{References}

1. Jemal A, Bray F, Center MM, Ferlay J, Ward E and Forman D: Global cancer statistics. CA Cancer J Clin 61: 69-90, 2011.

2. Cecilio AP, Takakura ET, Jumes JJ, Dos Santos JW, Herrera AC, Victorino VJ and Panis C: Breast cancer in Brazil: Epidemiology and treatment challenges. Breast Cancer (Dove Med Press) 7: 43-49, 2015.

3. Gonzaga CM, Freitas-Junior R, Curado MP, Sousa AL, Souza-Neto JA and Souza MR: Temporal trends in female breast cancer mortality in Brazil and correlations with social inequalities: Ecological time-series study. BMC Public Health 15: 96, 2015.

4. Polyak K: Heterogeneity in breast cancer. J Clin Invest 121: 3786-3788, 2011.

5. Martelotto LG, Ng CK, Piscuoglio S, Weigelt B and Reis-Filho JS: Breast cancer intra-tumor heterogeneity. Breast Cancer Res 16: $210,2014$.

6. Coates AS, Winer EP, Goldhirsch A, Gelber RD, Gnant M, Piccart-Gebhart M, Thürlimann B and Senn HJ; Panel Members: Tailoring therapies-improving the management of early breast cancer: St Gallen International Expert Consensus on the primary therapy of early breastcancer 2015. Ann Oncol26: 1533-1546,2015.
7. Cobleigh MA, Vogel CL, Tripathy D, Robert NJ, Scholl S, Fehrenbacher L, Wolter JM, Paton V, Shak S, Lieberman G and Slamon DJ: Multinational study of the efficacy and safety of humanized anti-HER2 monoclonal antibody in women who have HER2-overexpressing metastatic breast cancer that has progressed after chemotherapy for metastatic disease. J Clin Oncol 17: 2639-2648, 1999.

8. Early Breast Cancer Trialists' Collaborative Group (EBCTCG): Effects of chemotherapy and hormonal therapy for early breast cancer on recurrence and 15-year survival: An overview of the randomised trials. Lancet 365: 1687-1717, 2005.

9. Perou CM, Sørlie T, Eisen MB, van de Rijn M, Jeffrey SS, Rees CA, Pollack JR, Ross DT, Johnsen H, Akslen LA, et al: Molecular portraits of human breast tumours. Nature 406: 747-752, 2000.

10. Goldhirsch A, Wood WC, Coates AS, Gelber RD, Thürlimann B and Senn HJ and Panel members: Strategies for subtypes-dealing with the diversity of breast cancer: Highlights of the St. Gallen international expert consensus on the primary therapy of early breast cancer 2011. Ann Oncol 22: 1736-1747, 2011.

11. Yersal $\mathrm{O}$ and Barutca S: Biological subtypes of breast cancer: Prognostic and therapeutic implications. World J Clin Oncol 5: 412-424, 2014.

12. Paik S, Shak S, Tang G, Kim C, Baker J, Cronin M, Baehner FL, Walker MG, Watson D, Park T, et al: A multigene assay to predict recurrence of tamoxifen-treated, node-negative breast cancer. $\mathrm{N}$ Engl J Med 351: 2817-2826, 2004.

13. Allison KH: Molecular pathology of breast cancer: What a pathologist needs to know. Am J Clin Pathol 138: 770-780, 2012.

14. Prat A, Cheang MC, Martín M, Parker JS, Carrasco E, Caballero R, Tyldesley S, Gelmon K, Bernard PS, Nielsen TO and Perou CM: Prognostic significance of progesterone receptor-positive tumor cells within immunohistochemically defined luminal a breast cancer. J Clin Oncol 31: 203-209, 2013.

15. Maisonneuve P, Disalvatore D, Rotmensz N, Curigliano G, Colleoni M, Dellapasqua S, Pruneri G, Mastropasqua MG, Luini A, Bassi F, et al: Proposed new clinicopathological surrogate definitions of luminal A and luminal B (HER2-negative) intrinsic breast cancer subtypes. Breast Cancer Res 16: R65, 2014.

16. Subik K, Lee JF, Baxter L, Strzepek T, Costello D, Crowley P, Xing L, Hung MC, Bonfiglio T, Hicks DG and Tang P: The Expression Patterns of ER, PR, HER2, CK5/6, EGFR, Ki-67 and AR by immunohistochemical analysis in breast cancer cell lines. Breast Cancer (Auckl) 4: 35-41, 2010.

17. Wolff AC, Hammond ME, Hicks DG, Dowsett M, McShane LM, Allison KH, Allred DC, Bartlett JM, Bilous M, Fitzgibbons $\mathrm{P}$, et al: Recommendations for human epidermal growth factor receptor 2 testing in breast cancer: American society of clinical oncology/college of American pathologists clinical practice guideline update. Arch Pathol Lab Med 138: 241-256, 2014.

18. Sørlie T, Perou CM, Tibshirani R, Aas T, Geisler S, Johnsen H, Hastie T, Eisen MB, van de Rijn M, Jeffrey SS, et al: Gene expression patterns of breast carcinomas distinguish tumor subclasses with clinical implications. Proc Natl Acad Sci USA 98: 10869-10874, 2001.

19. Prat A, Parker JS, Karginova O, Fan C, Livasy C, Herschkowitz JI, He X and Perou CM: Phenotypic and molecular characterization of the claudin-low intrinsic subtype of breast cancer. Breast Cancer Res 12: R68, 2010.

20. Carey LA, Perou CM, Livasy CA, Dressler LG, Cowan D, Conway K, Karaca G, Troester MA, Tse CK, Edmiston S, et al: Race, breast cancer subtypes, and survival in the Carolina breast cancer study. JAMA 295: 2492-2502, 2006.

21. Ross JS and Fletcher JA: The HER-2/neu oncogene in breast cancer: Prognostic factor, predictive factor, and target for therapy. Oncologist 3: 237-252, 1998.

22. Cheang MC, Chia SK, Voduc D, Gao D, Leung S, Snider J, Watson M, Davies S, Bernard PS, Parker JS, et al: Ki67 index, HER2 status, and prognosis of patients with luminal B breast cancer. J Natl Cancer Inst 101: 736-750, 2009.

23. Cancer Genome Atlas Network: Comprehensive molecular portraits of human breast tumours. Nature 490: 61-70, 2012.

24. Holliday DL and Speirs V: Choosing the right cell line for breast cancer research. Breast Cancer Res 13: 215, 2011. 